\title{
Measurement of free surface energy of laser treated aluminum substrate
}

\author{
Kseniya Batishcheva ${ }^{1, *}$, Dmitriy Feoktistov ${ }^{1}$, Anastasiya Islamova ${ }^{1}$, and Ivan Shanenkov ${ }^{1}$ \\ ${ }^{1}$ National Research Tomsk Polytechnic University, 634050 Tomsk, Russia
}

\begin{abstract}
The change in the surface energy of the aluminum substrate after laser treatment has been determined experimentally. Surface energy is determined using the Advance KRUSS software. The laser treatment of the surface is found to increase its free surface energy. It changes the ratio of the polar and the dispersion components. Analysis of the crystalline planes of aluminum surface after laser treatment was provided by XRD X-ray diffractometry using a Shimadzu XRD 7000S diffractometer.
\end{abstract}

\section{Introduction}

Hydrophobic and hydrophilic materials are in demand in many spheres of human activity. The most of known methods both improving and deteriorating wettability of various materials [1-3] have a disadvantage. It is a limited period of use after which the surface acquires the initial wettability properties. Such changes occur under the influence of the surrounding aggressive environment or a simple mechanical abrasion of the surface. The development of a method for the surface properties changes due to transformations in the surface texture [4] is the most crucial topic nowadays. In addition, the period of use for such materials will significantly increase. One of these methods is laser radiation treatment.

It is known [5] that the behavior of a liquid drop on a solid surface depends on surface properties, such as surface and interfacial tension, surface free energy (SFE). The surface free energy can be found experimentally in determining the wetting angle of the surface by liquids having different polar and dispersion components. There are several scientific approaches for the definition of SFE. The most common are the following:

1. Zisman plot method [6]. From the experimental values of the contact angle $\theta$, a dependence is plotted in the axes $\cos \theta$ ( $\mathrm{Y}$ axis) $-\theta$ ( $\mathrm{X}$ axis), then extrapolation is used to determine the SFE. This method is used for wetting of non-polar surfaces since Zisman method does not take into account the intermolecular interaction between a liquid and a solid surface. In addition, it fails for high-energy samples where no linear trend in the plots was observed [7].

2. The Owens-Wendt-Rabel-Kaelble method (OVRK) $[8,9]$ differentiates polar and dispersion components of the surface energy and uses a geometric mean of these in the expression for it according to the following equation:

\footnotetext{
* Corresponding author: bka1801@mail.ru
} 


$$
\frac{\sigma_{L}(\cos \theta+1)}{2 \sqrt{\sigma_{L}^{D}}}=\frac{\sqrt{\sigma_{S}^{P}} \cdot \sqrt{\sigma_{L}^{P}}}{2 \sqrt{\sigma_{L}^{D}}}+\sqrt{\sigma_{S}^{D}}
$$

where $\sigma_{L}$ is surface tension of liquid; $\sigma_{S}$ surface free energy of a solid; $\sigma_{L}^{D}$ dispersion component of liquid; $\sigma_{S}^{D}$ dispersion component of surface; $\sigma_{L}^{P}$ polar component of liquid; $\sigma_{S}^{P}$ polar component of surface.

It is assumed that the van der Waals force has the greatest influence on the dispersion component of the solid, the interaction forces and hydrogen bonds - on the polar component.

3. Fowkes method [10] is based on theory of liquid adhesion and wetting of solid and used for determination of the SFE of polar surfaces.

4. Wu method [11]. The method is based on the equation:

$$
\sigma_{L}(\cos \theta+1)=4\left(\frac{\sigma_{S}^{D} \cdot \sigma_{L}^{D}}{\sigma_{S}^{D}+\sigma_{L}^{D}}+\frac{\sigma_{S}^{P} \cdot \sigma_{L}^{P}}{\sigma_{S}^{P}+\sigma_{L}^{P}}\right)
$$

It is used for solids with relatively large surface free energy [5].

5. van Oss and Good method [12] is based on equation:

$$
(1-\cos \theta) \cdot \sigma_{L}=\sigma_{S}+\sigma_{L}-2 \cdot\left(\sqrt{\sigma_{S}^{D} \cdot \sigma_{L}^{D}}+\sqrt{\sigma_{S}^{+} \cdot \sigma_{L}^{-}}+\sqrt{\sigma_{S}^{-} \cdot \sigma_{L}^{+}}\right)
$$

The difference from the above mentioned methods is in the assumption that the polar component of the SFE consists of acid and base parts.

An analysis of the use of the methods described above was made in [7]. The results of the SFE determination are found to depend on the test liquids chosen. The most accurate results were obtained using water and methylene diiodide. The OVRK method is recommended as the most accurate.

The aim of the work is to determine the effect of laser radiation on the change in the SFE of an aluminum surface.

\section{Materials and methods}

Experimental studies were carried out using a shadow optical system on a setup presented in Fig. 1 [13]. 


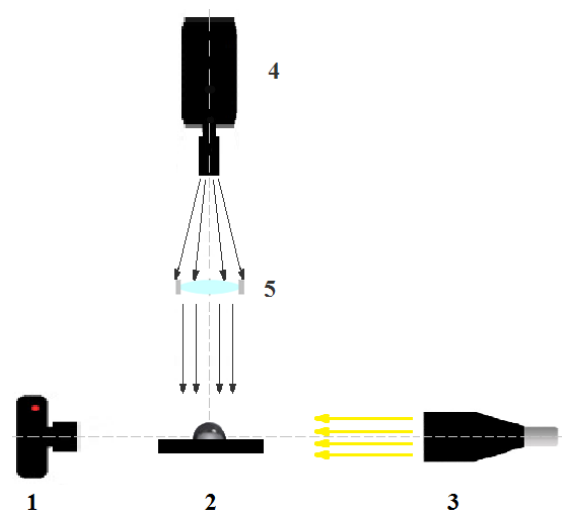

Fig. 1. Schematic of the experimental setup: 1 - photographic camera, 2 - substrate with a drop, 3 source of plane-parallel light, 4 - high-speed video camera, 5 - condensing lens.

A drop of distilled water (methylene diiodide) of $10 \mu \mathrm{L}$ was placed on an aluminum substrate (2) by high-precision dispenser and illuminated by a plane-parallel light (3), wherein a part of the light was dissipated by a drop, and non-dissipated light was fixed by the camera. Dash-dotted line shows the optical axis of the shadow system. Video camera (4) and condensing lens (5) were placed above the liquid drop and allowed visualization of evaporation process from the top and control of drop symmetry.

The experiment was carried out using four aluminum alloy (AMG-6) substrates. The surfaces were polished. After polishing, three substrates were treated with laser radiation, one was not processed. The texture is treated by a laser system "Mini Marker 2 M 20". Technical characteristics of the laser system used for surface treatment of aluminum experimental samples are listed in Table 1 .

Table 1. Technical characteristics of laser system used for surface treatment.

\begin{tabular}{|c|c|}
\hline Frequency of the laser beam, $\mathrm{Hz}$ & 99 \\
\hline Travel speed of the laser beam, $10^{-3} \mathrm{~m} / \mathrm{sec}$ & 800 \\
\hline Power of the laser radiation, $\mathrm{W}$ & $6,14,18$ \\
\hline
\end{tabular}

Surface energy is determined using the Advance KRUSS software.

The surfaces after laser treatment were studied by XRD X-ray diffractometry using a Shimadzu XRD 7000S diffractometer (Cuk $\alpha$ - radiation, $\lambda=1.540598$, with focusing on the secondary beam monochromator). Interpretation of the phase composition was carried out by processing the obtained diffractograms in the Search-Match software package using the PDF2 database.

\section{Results and discussion}

Figure 2 provides the diffractograms of samples treated by laser beam with different powers in comparison with the diffractogram of polished substrate. The dominant phase is expected to be pure aluminum. The intensity of maxima reduced with increasing the power of laser radiation (Fig. 2). It can be explained by etching a part of the samples surface and, correspondingly, by reducing the useful area of the sample which interacts with X-rays. This affects the reduction of integrated statistical information which causes a decrease in the intensity. The position of maxima on the $2 \theta$-axis refers to alumina phase (Fig. 2). The change of the preferential orientation of sample maxima obtained at the radiation power of $6 \mathrm{~W}$ is the most interesting. The explanation for this fact can be a change in the preferential 
orientation of the planes in the investigated subsurface layer. However, for the other two samples, the ratio of the maxima remained unchanged.

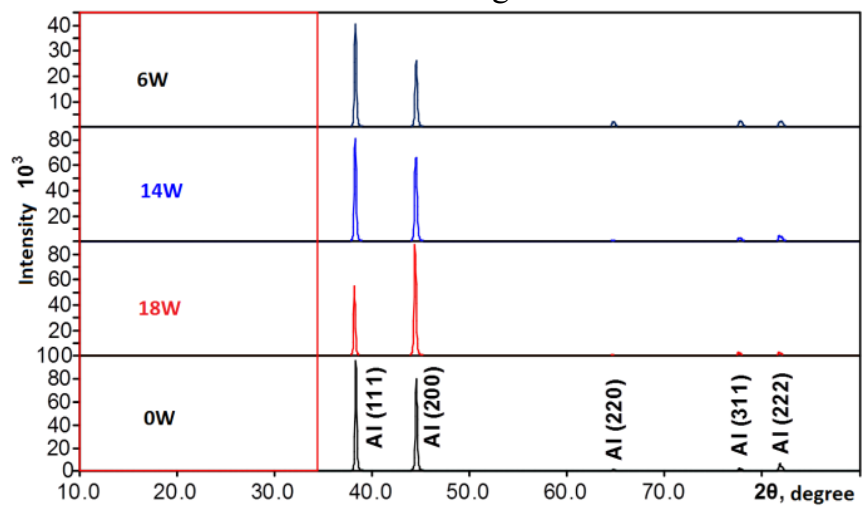

Fig. 2. Diffractograms of surfaces after laser radiation treating.

Figure 3 shows the diffractograms of surfaces in the region of small angles $2 \theta$ up to $35^{\circ}$.

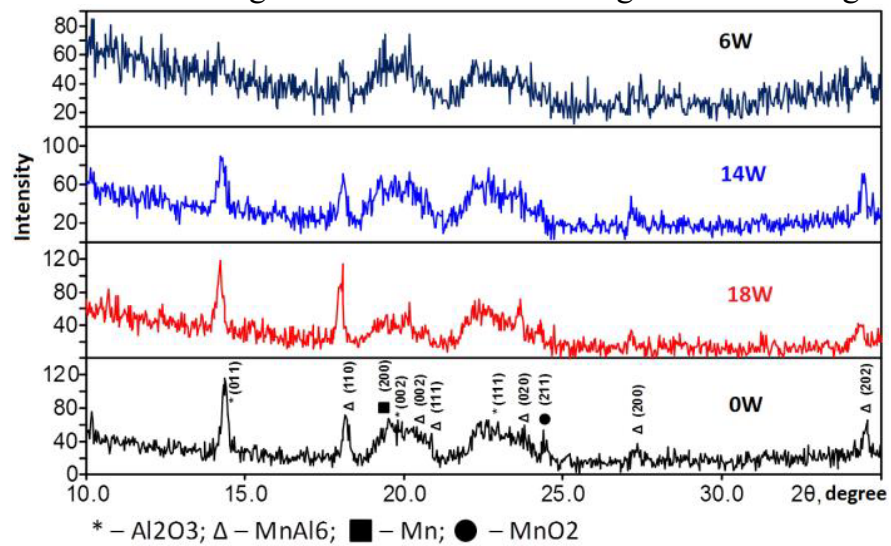

Fig. 3. Diffractograms of surfaces in the region of small angles.

According to the results presented in Fig. 3 it is found that there are reflections of crystal planes of the following phases: aluminum oxide $\mathrm{Al}_{2} \mathrm{O}_{3}$, aluminum and manganese alloy $\mathrm{MnAl}_{6}$, pure manganese $\mathrm{Mn}$, and manganese oxide $\mathrm{MnO}_{2}$. The presence of these impurities containing manganese is also explained by its presence in alloy composition. The presence of oxide phases is quite natural, such as aluminum is chemically active element and is easily oxidized in air. Based on diffractograms (Fig. 3) it is seen that with increasing laser beam power, the intensity and the number of maxima of the impurity phases decrease. Traces of other elements which are present in the alloy in large quantities were not found on diffractograms.

Figure 4 shows the results of determining the change of surface energy. 


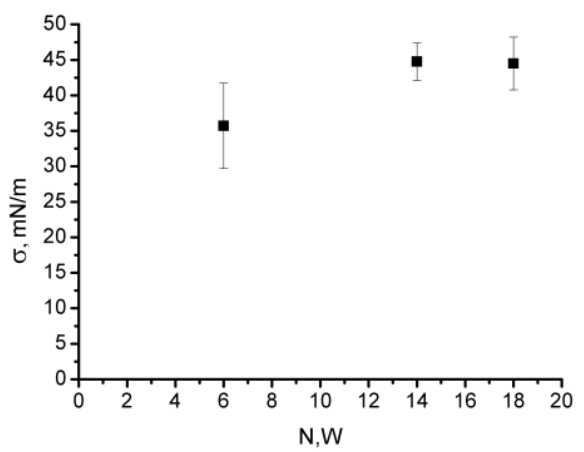

Fig. 4. Dependence of the surface energy on the laser beam power.

\section{Conclusions}

The influence of laser radiation on the change in the SFE of the aluminum surface is determined experimentally. The SFE is found to increase with increasing laser power. It changes the ratio of the polar and dispersion components. The proportion of the dispersion component increases. Analyses of the crystalline planes of aluminum surface after laser treatment was provided by XRD X-ray diffractometry using a Shimadzu XRD 7000S diffractometer (Cuk $\alpha$ - radiation, $\lambda=1.540598$, with focusing on the secondary beam monochromator). It is found that the laser surface treatment changes the orientation of the crystal planes. It should be noted that this result is obtained only at a relatively low power of the laser beam $(6 \mathrm{~W})$. The orientation of the crystalline planes of the aluminum surface did not change at high power of the laser.

The reported study was supported by the grant of President of Russian Federation for the government support of young Russian scientists (MK-6810.2016.8).

\section{References}

1. D. Zaitsev, D. Kirichenko, O. Kabov, Technical Physics Letters, 41, 6 (2015)

2. E.G.Orlova, E.R.Malyhina, D.V.Feoktistov, L.S.Zhidkova, MATEC Web Conf. 72, 01062 (2016)

3. Ya. Misyura, Appl. Surf. Sci. 414, 188 (2017)

4. D. V. Ta, A. Dunn, T. J. Wasley, R. W. Kay, J. Stringer, P. J. Smith, C. Connaughton, J. D. Shephar, Appl. Surf. Sci. 357, 248-254 ( 2015)

5. M. Tryznowski, J. Izdebska-Podsiadly, Z. Zolek-Tryznowska, Prog. Org. Coat. 109, 55-60 (2017)

6. H. Fox, W. Zisman, J. Colloid Sci. 5, 514-531, (1950)

7. F. Hejda, P. Solar, J. Kousal, WDS'10 Proceedings of Contributed Papers, Part III, 25 (2010)

8. D. Owens, R. Wendt, J. Appl. Polym. Sci. 13, 1741 (1969)

9. D. Kaelble, J. Adhes. 2, 66 (1970)

10. S. Fowkes, J. Phys. Chem. 67, 2538 (1963)

11. S. Wu, J. Polym. Sci. C 34, 19 (1971)

12. C. van Oss, M. Chadhury, R. Good, Adv. Colloid Interface Sci. 28, 35 (1987)

13. G. Kuznetsov, D. Feoktistov, E. Orlova, K. Batishcheva, Colloid J. 78, 3 (2016) 